\title{
Nonimmune hydrops fetalis: identifying the underlying genetic etiology
}

\author{
Teresa N. Sparks, MD ${ }^{1,2}$, Kao Thao, BA ${ }^{1}$, Billie R. Lianoglou, LCGC ${ }^{1,2}$, Nina M. Boe, MD ${ }^{3}$, \\ Kari G. Bruce, $\mathrm{MD}^{4}$, Ilina Datkhaeva, $\mathrm{MD}^{5}$, Nancy T. Field, $\mathrm{MD}^{3}$, Victoria M. Fratto, $\mathrm{MD}^{6}$, \\ Jennifer Jolley, MD, Louise C. Laurent, MD, $\mathrm{PhD}^{6}$, Anne H. Mardy, MD ${ }^{1}$, Aisling M. Murphy, MD ${ }^{5}$, \\ Emily Ngan, MD ${ }^{6}$, Naseem Rangwala, BA ${ }^{1}$, Catherine A. M. Rottkamp, MD, PhD ${ }^{8}$, Lisa Wilson, BS ${ }^{1}$, \\ Erica $\mathrm{Wu}, \mathrm{MD}^{4}$, Cherry C. Uy, , MD ${ }^{4}$, Priscila Valdez Lopez, $\mathrm{BS}^{1}$ and \\ Mary E. Norton, $\mathrm{MD}^{1,2}$ on behalf of the University of California Fetal-Maternal Consortium (UCfC)
}

Purpose: Numerous etiologies may lead to nonimmune hydrops fetalis (NIHF), and the underlying cause often remains unclear. We aimed to determine the proportion of NIHF cases in which the etiology was clearly determined in a large, contemporary, and diverse cohort, as well as to describe the etiologies with a focus on genetic causes.

Methods: Retrospective review of NIHF cases between 2015 and 2017 from the five University of California Fetal-Maternal Consortium sites. Singleton pregnancies with prenatally diagnosed NIHF were included, and cases with maternal alloimmunization were excluded. Cases were categorized as being of confirmed, suspected, or unknown etiology.

Results: Sixty-five NIHF cases were identified. Forty-six percent $(30 / 65)$ remained of unknown etiology, while 9.2\% (6/65) had a suspected etiology and $44.6 \%$ (29/65) were of confirmed etiology.
Among confirmed cases, 11 resulted from aneuploidy; 7 from fetal structural anomalies; 2 each from fetal arrhythmia, Noonan syndrome, and generalized lymphatic dysplasia; and 1 each from arthrogryposis, parvovirus, neonatal alloimmune thrombocytopenia, fetal goiter, and Kasabach-Merritt syndrome.

Conclusion: In this contemporary, multicenter study, the cause of prenatally diagnosed NIHF was confirmed in only $44 \%$ of cases, and a genetic etiology was found in only $25 \%$ of those that received standard of care genetic testing.

Genetics in Medicine (2019) 21:1339-1344; https://doi.org/10.1038/s41436018-0352-6

Keywords: etiology; diagnostic evaluation; genetic; hydrops fetalis; nonimmune

\section{INTRODUCTION}

Hydrops fetalis affects between 1 in 1700 and 1 in 3000 pregnancies, and is associated with significant perinatal complications ranging from preterm birth to mirror syndrome, stillbirth, and neonatal demise. ${ }^{1-6}$ Hydrops is diagnosed by sonographic detection of two or more abnormal fetal fluid collections, including ascites, pericardial effusion, pleural effusion, or skin edema. ${ }^{1}$ With appropriate $\mathrm{Rh}(\mathrm{D})$ immune globulin administration, only $10 \%$ of hydrops cases are attributable to alloimmunization, and the remainder result from a wide variety of nonimmune etiologies such as chromosomal abnormalities, inborn errors of metabolism, infections, ${ }^{6}$ and fetal structural anomalies. ${ }^{1,7-11}$
The Society for Maternal-Fetal Medicine has published recommendations for the diagnostic evaluation of nonimmune hydrops fetalis (NIHF) that include obstetric ultrasound, fetal echocardiogram, fetal karyotype and/or chromosomal microarray analysis (CMA), viral polymerase chain reaction (PCR), and consideration of more specialized testing. ${ }^{1}$ Even with these evaluations, the etiology remains unclear in a substantial proportion of cases, with reports ranging from $20 \%$ to $68 \%$ (refs. ${ }^{1,8,9}$ ). In particular, limited data have been published on the yield of CMA for NIHF since it was recommended in place of karyotype for diagnostic testing of fetal anomalies, ${ }^{12}$ and next steps for investigation beyond the standard genetic workup are not well defined. For example, $19 \%$ of all NIHF cases have been attributed to rare

\footnotetext{
${ }^{1}$ Department of Obstetrics, Gynecology, \& Reproductive Sciences, University of California, San Francisco, CA, USA; ${ }^{2}$ Fetal Treatment Center, University of California, San Francisco, CA, USA; ${ }^{3}$ Department of Obstetrics \& Gynecology, University of California, Davis, CA, USA; ${ }^{4}$ Department of Pediatrics, University of California, Irvine, CA, USA; ${ }^{5}$ Department of Obstetrics \& Gynecology, University of California, Los Angeles, CA, USA; ${ }^{6}$ Department of Obstetrics, Gynecology, and Reproductive Biology, University of California, San Diego, CA, USA; ${ }^{7}$ Department of Obstetrics \& Gynecology, University of California, Irvine, CA, USA; ${ }^{8}$ Department of Pediatrics, University of California, Davis, CA, USA. Correspondence: Teresa N. Sparks (teresa.sparks@ucsf.edu)
} 
genetic syndromes and nearly one-third of idiopathic cases to lysosomal storage disorders (LSDs), ${ }^{7,13}$ which are not detected through karyotype or CMA. Because the majority of existing literature consists of case reports and small series focusing on specific genetic diseases, ${ }^{14-25}$ the true frequency of each underlying cause remains uncertain.

Without a better understanding of the causes of NIHF, it is challenging to effectively manage these pregnancies, counsel about prognosis and recurrence risk, and anticipate neonatal care requirements. Our objectives were to determine the proportion of NIHF cases in which the etiology was clearly determined in a large, contemporary, and diverse cohort for which providers followed current guidelines for diagnostic testing, as well as to describe the etiologies for confirmed cases with a focus on the genetic causes. We hypothesized that a substantial proportion of NIHF cases would remain of unclear etiology, and that chromosomal abnormalities and congenital anomalies would underlie most cases with a confirmed etiology.

\section{MATERIALS AND METHODS}

This was a retrospective multicenter review of all NIHF cases evaluated through the University of California Fetal_Maternal Consortium (UCfC) between January 2015 and April 2017. The UCfC is a collaborative network that was designed to investigate and implement best clinical practices across the University of California campuses (UC Davis, UC Irvine, UC Los Angeles, UC San Diego, and UC San Francisco). Multiinstitutional review board reliance registry approval was obtained (Institutional Review Board No. 10-04093). Because of the retrospective nature and approval of this study, informed consent was not required from participants.

Inclusion criteria were prenatally diagnosed NIHF cases. Exclusion criteria were multiple gestations and cases of hydrops resulting from maternal alloimmunization. The primary outcome was the proportion of prenatally diagnosed NIHF cases in which the underlying etiology was clearly confirmed. Secondary outcomes were type of etiology for cases in which a diagnosis was confirmed, as well as specific genetic etiologies identified with the current approach to testing.

The primary and senior authors (T.N.S. and M.E.N.) categorized cases into groups of confirmed etiology, suspected etiology, or unknown etiology. Cases were categorized as confirmed when a clearly defined underlying disease process or structural anomaly was identified that is strongly supported by the existing literature as leading to NIHF. Examples of confirmed etiologies were Down syndrome, congenital diaphragmatic hernia, and Noonan syndrome. Cases were categorized as suspected when there was either supporting but inconclusive literature about the potential for the disease process leading to NIHF, or there was a lack of diagnostic testing to confirm the underlying diagnosis. Examples of suspected cases were those with positive titers for viruses potentially capable of causing NIHF, or a screen positive result for aneuploidy without diagnostic testing. When there was a lack of supporting literature to categorize cases as having either a confirmed or suspected NIHF etiology, or when the evaluation did not yield findings supportive of a specific underlying disease process, cases were categorized as being of unknown etiology. Examples of unknown cases were those with normal fetal anatomy and genetic testing, nonspecific structural anomalies such as ventricular septal defects, structural anomalies that do not clearly lead to NIHF such as aortic arch hypoplasia, and other nonspecific findings such as white matter defects.

Confirmed and suspected cases were further grouped into subcategories based on the specific underlying etiology. Cases in which aneuploidy was identified in addition to a fetal structural anomaly were grouped into the chromosomal abnormality category, with the rationale that the underlying aneuploidy explained the fetal structural abnormality as well as the manifestation of hydrops. The same rationale was applied to cases in which a pathogenic genetic variant was identified in addition to a fetal structural anomaly.

Physicians and research assistants at each site collected data through chart review, and entered this data into a REDCap database. Data collected included parental demographic data; family history including stillbirths, childhood deaths, and genetic syndromes; prenatal course, ultrasound findings, and evaluation performed; delivery data; and neonatal outcomes including additional testing performed after birth as well as survival to discharge. Details of genetic testing were collected, including the type of test as well as specific findings. Pregnancy outcomes were categorized as living at discharge, neonatal death, stillbirth (intrauterine fetal demise at $\geq 20$ weeks), spontaneous pregnancy loss (intrauterine fetal demise at $<20$ weeks), and termination. Infants and their outcomes were followed at each site until discharge from the hospital after birth. Due to the nature of our referral population, we did not have information about final pregnancy outcome data for ten cases in which women delivered at an outside institution or were otherwise lost to follow up. Additionally, a full NIHF evaluation was not completed in some cases when the decision was made to terminate the pregnancy, stillbirth occurred, or hydrops was diagnosed late in gestation. These cases were still included in our cohort though, to accurately reflect the diversity of the NIHF population seen at our institutions during the study time period.

We calculated the proportion of NIHF cases in which the etiology was confirmed after evaluation, suspected, or unknown. For confirmed and suspected cases, the proportions due subcategories of underlying etiologies were calculated. Fisher's exact test was used to compare proportions, and Wilcoxon rank sum test compared median values for nonparametric variables after data were evaluated for normalcy. A two-sided $p$ value of 0.05 was considered statistically significant, and STATA software (version 15.0, College Station, TX) was utilized for statistical analyses. 


\section{RESULTS}

A total of 65 NIHF cases were identified. Of these, $10.8 \%$ were from UC Davis, $18.5 \%$ from UC Irvine, $21.5 \%$ from UC Los Angeles, 23.1\% from UC San Diego, and 26.2\% from UC San Francisco. Table 1 outlines the demographics of the overall cohort. Three women had a prior pregnancy resulting in stillbirth, and one of these women had two prior pregnancies resulting in stillbirth. One stillbirth occurred in the setting of NIHF and congenital pulmonary airway malformation (CPAM), and one was thought due to placental abruption. For the woman who had two prior stillbirths, one was attributed to generalized lymphatic dysplasia with NIHF as described below, while the other did not have extensive evaluation and remained of unknown etiology. One woman had a prior pregnancy with a fetal structural anomaly (the previously mentioned CPAM).

Four women had a prior pregnancy with NIHF. One was attributed to $\alpha$-thalassemia, one to the previously mentioned $\mathrm{CPAM}$, and one to a large fetal goiter. The fourth woman had

Table 1 Demographics of the overall cohort

\begin{tabular}{|c|c|c|}
\hline \multicolumn{2}{|l|}{ Demographic } & Value $^{a}$ \\
\hline \multicolumn{3}{|c|}{ Personal and family history } \\
\hline \multirow[t]{3}{*}{ Personal history ${ }^{\mathrm{b}}$} & $\mathrm{NIHF}$ & $6.9 \%(4 / 58)$ \\
\hline & Stillbirth & $5.4 \%(3 / 56)$ \\
\hline & Fetal structural anomaly & $1.9 \%(1 / 53)$ \\
\hline \multirow[t]{4}{*}{ Family history ${ }^{\mathrm{b}}$} & $\mathrm{NIHF}$ & $1.9 \%(1 / 54)$ \\
\hline & Stillbirth & $5.6 \%(3 / 54)$ \\
\hline & Fetal structural anomaly & $1.8 \%(1 / 56)$ \\
\hline & Consanguinity & $3.9 \%(2 / 51)$ \\
\hline \multicolumn{3}{|l|}{ Race/ethnicity } \\
\hline \multirow[t]{5}{*}{ Maternal race ${ }^{c}$} & Asian & $15.7 \%(8 / 51)$ \\
\hline & Hawaiian/Other PI & $2.0 \%(1 / 51)$ \\
\hline & White & $74.5 \%(38 / 51)$ \\
\hline & Other & $3.9 \%(2 / 51)$ \\
\hline & Multiracial & $3.9 \%(2 / 51)$ \\
\hline Maternal ethnicity ${ }^{d}$ & Hispanic/Latina & $43.1 \%(25 / 58)$ \\
\hline \multicolumn{3}{|c|}{ Current pregnancy with NIHF } \\
\hline \multicolumn{2}{|l|}{ Maternal age (years) } & $29.0(16-43)$ \\
\hline \multicolumn{2}{|c|}{ Gestational age at hydrops diagnosis (weeks) } & $22.0(11.3-37.4)$ \\
\hline \multicolumn{2}{|c|}{ Gestational age at delivery (weeks) ${ }^{\mathrm{e}}$} & $33.2(25.6-39.4)$ \\
\hline \multirow[t]{5}{*}{ Pregnancy outcome } & Live infant & $25.5 \%(14 / 55)$ \\
\hline & Infant demise & $29.1 \%(16 / 55)$ \\
\hline & Stillbirth & $20.0 \%(11 / 55)$ \\
\hline & Spontaneous abortion & $5.5 \%(3 / 55)$ \\
\hline & Termination & $20.0 \%(11 / 55)$ \\
\hline
\end{tabular}

NIHF nonimmune hydrops fetalis, PI Pacific Islander.

${ }^{a}$ Median values (with ranges) shown for continuous variables, percent $(N)$ shown for proportions.

${ }^{b}$ Excluding cases for which this history was unknown. Family history was entered once for one woman who had two hydropic pregnancies.

${ }^{\text {c}}$ Thirteen cases were of unknown race or declined to answer. Race was entered once for one woman who had two hydropic pregnancies. There were no women of American Indian or Black race.

${ }^{d}$ Six cases were of unknown ethnicity or declined to answer. Ethnicity was entered once for one woman who had two hydropic pregnancies.

excluding terminations, spontaneous losses, and stillbirths a prior pregnancy with generalized lymphatic dysplasia and NIHF as mentioned previously. This was discovered after exome sequencing in her subsequent pregnancy that was also affected by NIHF revealed two likely pathogenic PIEZO1 variants as outlined below.

In terms of overall NIHF evaluation and yield of tests performed, all cases in our cohort had an obstetric ultrasound demonstrating hydrops. Eleven percent (7/65) were found to have a concurrent structural anomaly that clearly explained the presentation of NIHF. Sixty percent (39/65) had a fetal echocardiogram, $4(10.3 \%, 4 / 39)$ of which showed a cardiac abnormality that led to a confirmed etiology of NIHF. Hemoglobin electrophoresis was done in $10.8 \%$ (7/65) of cases and Kleihauer-Betke test was done in 9.2\% (6/65); all were normal. Forty-three percent (28/65) of cases had middle cerebral artery (MCA) Dopplers done, $25.0 \%$ (7/28) of which were elevated. Finally, 50.8\% (33/65) had an infectious workup for parvovirus, toxoplasmosis, cytomegalovirus, herpes virus, syphilis, and/or other viruses. In 9\% (3/33) of these cases, infectious testing yielded a confirmed or suspected diagnosis as further discussed below. Many of the cases lacking these evaluations were terminated or had resulted in a stillbirth.

Overall, 67.7\% (44/65) of the cohort received standard of care genetic testing with CMA and/or karyotype. Of these, $25.0 \%(11 / 44)$ had a result leading to a confirmed etiology of NIHF. The addition of exome sequencing or targeted genetic sequencing led to diagnosis in 4 additional cases. Table 2 outlines the genetic testing performed for evaluation of cases in our cohort. Forty cases had a karyotype, 11 of which were abnormal and showed Turner syndrome, trisomy 21, trisomy 18 , and a chromosome 2 inversion. The chromosome 2 inversion was a balanced paracentric inversion reported as 46 , $\mathrm{XY}$,inv(2)(q?14.2q23), and due to lack of parental karyotype data, was assessed as uncertain if de novo or inherited and as not clearly causative of the NIHF. Thirty cases had a CMA, 2 of which were abnormal (both showing trisomy 21). One of these cases also had a karyotype consistent with trisomy 21, and the other only had a CMA sent. Targeted PTPN11 sequencing was performed in two cases, showing pathogenic variants (c.417G $>$ C, c. $317 \mathrm{~A}>\mathrm{C}$ ) that led to a diagnosis of Noonan syndrome. A hydrops gene panel was sent in two cases, a RASopathy gene panel in three, and a lysosomal storage enzyme assay panel in one, all of which yielded normal results.

Exome sequencing was performed in two cases, yielding abnormal results for one: compound heterozygous PIEZO1 variants reported as likely pathogenic (c.3206G $>$ A of paternal inheritance, and c.6208A $>C$ of maternal inheritance). These were predicted to result in a nonsense (p.Trp1069*) change not tolerated in six prediction algorithms and a missense ( $\mathrm{p}$. Lys2070Gln) change not tolerated five of six prediction algorithms, respectively. The PIEZO1 protein regulates electrical currents and is important in the development of the lymphatic system and in red blood cell morphology. These exact variants have not previously been described, but loss-of- 
Table 2 Genetic workup performed for all cases of NIHF

\begin{tabular}{|c|c|c|c|}
\hline Test & $\begin{array}{l}\text { Number } \\
\text { cases } \\
\text { receiving } \\
\text { test }\end{array}$ & $\begin{array}{l}\text { Number } \\
\text { cases with } \\
\text { abnormal } \\
\text { result }\end{array}$ & $\begin{array}{l}\text { Abnormal findings } \\
\text { (number of cases } \\
\text { with each finding) }\end{array}$ \\
\hline Karyotype & 40 & 11 & $\begin{array}{l}\text { Turner syndrome (6), } \\
\text { trisomy } 21 \text { (3), } \\
\text { trisomy } 18 \text { (1), } \\
\text { chromosome } 2 \\
\text { inversion (1) }\end{array}$ \\
\hline $\begin{array}{l}\text { Chromosomal } \\
\text { microarray }\end{array}$ & 30 & 2 & Trisomy 21 (2) \\
\hline $\begin{array}{l}\text { Exome } \\
\text { sequencing }\end{array}$ & 2 & 1 & $\begin{array}{l}\text { Compound } \\
\text { heterozygous PIEZO1 } \\
\text { variants }(1)^{\mathrm{a}}\end{array}$ \\
\hline $\begin{array}{l}\text { Targeted } \\
\text { genetic testing }\end{array}$ & 3 & 3 & $\begin{array}{l}\text { PTPN11 pathogenic } \\
\text { variant (2), } \\
\text { Compound } \\
\text { heterozygous PIEZO1 } \\
\text { variants }(1)^{\mathrm{a}}\end{array}$ \\
\hline $\begin{array}{l}\text { Hydrops gene } \\
\text { panel }\end{array}$ & 2 & 0 & - \\
\hline $\begin{array}{l}\text { RASopathy } \\
\text { gene panel }\end{array}$ & 3 & 0 & - \\
\hline $\begin{array}{l}\text { Lysosomal } \\
\text { storage } \\
\text { enzyme assay } \\
\text { panel }\end{array}$ & 1 & 0 & - \\
\hline \multicolumn{4}{|c|}{$\begin{array}{l}\text { aExome sequencing identified compound heterozygous PIEZO1 variants } \\
\text { (c.3206G }>A \text { of paternal inheritance, and c. } 6208 \mathrm{~A}>\mathrm{C} \text { of maternal inheritance) in } \\
\text { the second hydropic fetus for one woman. Remaining DNA from the first hydro- } \\
\text { pic fetus was sequenced, and found to have the same compound heterozygous } \\
\text { PIEZO1 variants. } \\
\text { bPathogenic variants identified in PTPN11 were } C .417 \mathrm{G}>\mathrm{C} \text { for one case, and } \\
\text { c.317A }>C \text { for the other } \\
\text { CMA chromosomal microarray analysis, NIHF nonimmune hydrops fetalis. }\end{array}$} \\
\hline
\end{tabular}

function PIEZO1 variants have been implicated in generalized lymphatic dysplasia. ${ }^{26,27}$ This case was the later sibling of another NIHF case in our cohort, which had resulted in a stillbirth. Targeted sequencing was then performed on DNA remaining from the prior fetus, revealing the same two PIEZO1 variants.

Forty-six percent (30/65) of NIHF cases in our cohort remained of unknown etiology, while 9.2\% (6/65) had a suspected but unconfirmed etiology and $44.6 \%(29 / 65)$ had a confirmed etiology. Excluding the 10 cases for which outcome data were unavailable, these proportions did not change substantially: $45.5 \%$ (25/55) remained of unknown etiology, $7.3 \%(4 / 55)$ were of suspected etiology, and $47.3 \%(26 / 55)$ were of confirmed etiology.

Table 3 shows the etiologies underlying the confirmed and suspected cases. Among confirmed cases, etiologies in overall descending order of frequency included aneuploidy, fetal structural anomaly, and equal proportions of fetal arrhythmia, RASopathy, and generalized lymphatic dysplasia. Least
Table 3 Confirmed and suspected causes of NIHF

\begin{tabular}{lll} 
Etiology & & Percent $(N)$ \\
\hline Confirmed $(N=29)$ & Aneuploidy & $37.9 \%(11 / 29)$ \\
& Fetal structural anomaly & $24.1 \%(7 / 29)$ \\
& Fetal arrhythmia & $6.9 \%(2 / 29)$ \\
& RASopathy (Noonan syndrome) & $6.9 \%(2 / 29)$ \\
& Generalized lymphatic dysplasia & $6.9 \%(2 / 29)$ \\
& Arthrogryposis & $3.5 \%(1 / 29)$ \\
& Infectious (parvovirus) & $3.5 \%(1 / 29)$ \\
& Neonatal alloimmune thrombocytopenia & $3.5 \%(1 / 29)$ \\
& Fetal goiter & $3.5 \%(1 / 29)$ \\
Suspected $(N=6)$ & $3.5 \%(1 / 29)$ \\
& Kasabach-Merritt syndrome & $50.0 \%(3 / 6)$ \\
& Aneuploidy & $33.3 \%(2 / 6)$ \\
& Infectious (Coxsackie virus) & $16.7 \%(1 / 6)$ \\
\hline
\end{tabular}

NIHF nonimmune hydrops fetalis

frequent were one case each attributed to arthrogryposis, parvovirus, neonatal alloimmune thrombocytopenia, fetal goiter, and Kasabach-Merritt syndrome. Aneuploidies identified included Turner syndrome, trisomy 21, and trisomy 18 . Fetal structural anomalies included congenital diaphragmatic hernia $(\mathrm{CDH}), \mathrm{CPAM}$, intracranial mass resulting in hydrocephalus, and large cardiac mass. Fetal arrhythmias included fetal supraventricular tachycardia (SVT) and fetal heart block resulting from maternal systemic lupus erythematosus. The RASopathies identified were both Noonan syndrome. The one case resulting from an infectious etiology was attributed to parvovirus. The case of neonatal alloimmune thrombocytopenia (NAIT) was associated with an intracranial hemorrhage in utero and very preterm delivery due to nonreassuring fetal heart tracing. The case of Kasabach-Merritt syndrome was associated with large liver hemangioendothelioma, disseminated intravascular coagulopathy, and sagittal sinus thrombosis.

Among suspected cases, etiologies in descending order of frequency included aneuploidy, infectious, and hemoglobinopathy. Suspected aneuploidies included Turner syndrome and trisomy 21, which had been identified on prenatal genetic screening but were not confirmed with diagnostic testing. The two cases of infectious etiology were suspected to result from Coxsackie virus. One additional case occurred in a woman whose prior pregnancy was affected by $a$-thalassemia, but she had declined workup during the current pregnancy with NIHF.

Among the cases of unknown etiology, 60.0\% (18/30) of fetuses had one or more structural anomaly, while $40.0 \%$ $(12 / 30)$ were structurally normal. Undiagnosed cases with structural anomalies had anomalies that were assessed as not clearly causative or not typically associated with NIHF, such as cardiomyopathy, cardiac septal defects and other cardiac anomalies, renal agenesis, myelomeningocele, absent cavum septum pellucidum, cerebral ventriculomegaly, and talipes. One of the structurally normal cases had subcortical white matter defects and lactic acidemia, with presentation concerning for an inborn error of metabolism.

The median gestational age at delivery was 33.2 weeks (25.6-39.4) for the cases with continuing pregnancies. Sixtysix percent of cases with continuing pregnancies were 
delivered via cesarean. Near statistically significant differences were observed in pregnancy outcomes among confirmed cases when examined by category of etiology $(p=0.05)$. Among the eight cases of aneuploidy with a known pregnancy outcome, four resulted in stillbirth, one in spontaneous pregnancy loss, and three in termination. Of the seven cases with a fetal structural anomaly, two resulted in a living neonate at discharge (CPAM and $\mathrm{CDH})$, two resulted in neonatal demise (both $\mathrm{CDH}$ ), and three in termination (intracranial mass, cardiac mass, and CPAM). Among the two cases of fetal arrhythmia (fetal SVT and heart block due to systemic lupus erythematosus), both resulted in a living neonate at discharge. Of the two cases of Noonan syndrome, one was living at discharge and one was terminated. Of the two cases of generalized lymphatic dysplasia, one resulted in stillbirth and the other in neonatal demise. The case due to arthrogryposis resulted in stillbirth. The one case each due to Kasabach-Merritt syndrome and NAIT resulted in neonatal demise. The one case due to parvovirus resulted in a living neonate at discharge, as did the case due to fetal goiter.

\section{DISCUSSION}

In this multicenter, diverse, contemporary cohort of NIHF cases, we found that $46 \%$ of cases remained of unknown etiology, while $9 \%$ had a suspected but unconfirmed etiology and only $44 \%$ received a clear diagnosis. Specifically, a genetic etiology of NIHF was clearly found in $25 \%$ of the cohort that received standard of care genetic testing with CMA and/or karyotype. Among all cases of confirmed NIHF etiology, the most common were aneuploidy, fetal structural anomalies, fetal arrhythmias, Noonan syndrome, and generalized lymphatic dysplasia.

The proportion of cases in our study receiving a clear diagnosis for the cause of NIHF is somewhat smaller than in other series, although a wide range has been published. , $^{1,-9,28}$ We carefully reviewed the details of each NIHF case, and when there was a lack of clear or confirmatory data to support an etiology of the NIHF, cases were categorized as being of suspected or unknown etiology. The proportion of cases with a confirmed diagnosis in our study, as well as in others, may nevertheless be inflated. For example, while NIHF can be seen in the setting of $\mathrm{CDH}$ and Down syndrome, not all cases of $\mathrm{CDH}$ or Down syndrome develop hydrops. There may be additional factors such as genetic or epigenetic modifiers contributing to this more severe presentation in some cases. Thus, while we categorized cases of $\mathrm{CDH}$ and Down syndrome as being of confirmed etiology, the lack of more extensive genetic and other testing may miss underlying factors leading to a more severe phenotype with hydrops.

That nearly half of NIHF cases remain of unknown etiology highlights both our lack of understanding of the pathologic mechanisms leading to hydrops, as well as the incomplete nature of our current approach to evaluation. Further, while recommendations have been published for evaluation of NIHF cases, ${ }^{1}$ our understanding of what constitutes an adequate workup is currently limited. The wide diversity of conditions leading to hydrops, and the rarity of many of these conditions, lead to difficulty in confirming a diagnosis. While standard karyotype and CMA can identify some genetic abnormalities, these tests do not detect variants in exonic or intronic regions of the genome. Many genetic syndromes that can present with NIHF are caused by variants not detected by karyotype or CMA, such as inborn errors of metabolism and rare autosomal recessive conditions..$^{9,11,13-25}$ In addition, the yield of CMA for NIHF appears to be limited, both in terms of diagnostic yield and in achieving a diagnosis beyond that detectable by karyotype. Only $25 \%$ of the cohort that received a CMA or karyotype had a result leading to a confirmed etiology of NIHF, and CMA did not yield any findings that would have been missed by karyotype. A more expansive and evidence-based approach to the genetic workup in cases of NIHF is warranted, and further research will be needed to refine our current testing algorithm.

The strengths of this study include that it is among the largest series to date of prenatally diagnosed NIHF cases, and is based upon a diverse multicenter patient population. It also represents a contemporary cohort of NIHF cases, which is important in the setting of evolving genetic testing for such cases as well as improving technologies for prenatal ultrasound diagnosis. Further, this is one of few studies to focus on the genetic testing approach to workup of NIHF. However, this study is not without limitations. Although this cohort is relatively large compared with other published series, its overall size for the purpose of statistical analyses is somewhat small, which may have limited our ability to detect differences in outcomes among subgroups. Due to its retrospective nature, some information was more difficult to obtain or unable to be obtained, such as details of family history and racial/ethnic background. Because each of the UCfC sites is a major referral center, some women ultimately resumed care or delivered at outside facilities, which limited our ability to obtain follow-up data for these cases. Additionally, infant follow-up data was not available beyond initial discharge from the hospital.

In summary, a clear etiology of NIHF was determined in fewer than half of the cases in our multicenter cohort. Further, standard genetic testing led to a clearly established diagnosis in only one quarter of cases receiving this testing. Due to the heterogeneity in etiologies that may underlie NIHF, a broad and evidence-based approach to evaluation is necessary. This study points to the need for further research using more expansive genetic, infectious, and other testing to accurately determine the etiology of NIHF, as well as to the need for research to clarify the optimal algorithm for evaluation.

\section{ACKNOWLEDGEMENTS}

T.N.S. is supported by grant $5 \mathrm{~K} 12 \mathrm{HD} 001262-18$ from the National Institutes of Health $(\mathrm{NIH})$. The contents of the publication are solely the responsibility of the authors and do not necessarily represent the official views of the $\mathrm{NIH}$. We thank all members of the University of California Fetal-Maternal Consortium (UCfC), as well as the members of the Center for Maternal-Fetal Precision 
Medicine at UCSF, for their contributions to and support of this project. The abstract for this paper was presented in poster format (abstract \#437) at the 38th Society for Maternal-Fetal Medicine Meeting, which took place from 29 January to 3 February 2018 in Dallas, Texas.

\section{DISCLOSURE}

Ultragenyx has provided financial support for studies conducted through the UCSF Center for Maternal-Fetal Precision Medicine. M.E.N. is a consultant to Invitae and has received research funding from Natera, but this funding was not applied to this study. The other authors declare no conflicts of interest.

\section{REFERENCES}

1. Norton ME, Chauhan SP, Dashe JS. Society for Maternal-Fetal Medicine (SMFM) clinical guideline \#7: nonimmune hydrops fetalis. Am J Obstet Gynecol. 2015;212:127-139.

2. Derderian SC, Jeanty C, Fleck SR, et al. The many faces of hydrops. J Pediatr Surg. 2015;50:50-54.

3. Berger VK, Sparks TN, Jelin AC, et al. Non-immune hydrops fetalis: do placentomegaly and polyhydramnios matter? J Ultrasound Med. 2018;37:1185-1191.

4. Steurer MA, Peyvandi S, Baer RJ, et al. Epidemiology of live born infants with non-immune hydrops fetalis-insights from a population-based cohort. J Pediatr. 2017;187:182-188.

5. Gedikbasi A, Oztarhan K, Gunenc Z, et al. Preeclampsia due to fetal nonimmune hydrops: Mirror syndrome and review of the literature. Hypertens Pregnancy. 2011;30:322-330.

6. Fukushima K, Morokuma S, Fujita $Y$, et al. Short-term and long-term outcomes of 214 cases of non-immune hydrops fetalis. Early Hum Dev. 2011;87:571-575.

7. Moreno CA, Kanazawa T, Barini R, et al. Non-immune hydrops fetalis: a prospective study of 53 cases. Am J Med Genet A. 2012;161:3078-3086.

8. Santo S, Mansour S, Thilaganathan B, et al. Prenatal diagnosis of nonimmune hydrops fetalis: what do we tell the parents? Prenat Diagn. 2011;31:186-195.

9. Bellini C, Donarini G, Paladini D, et al. Etiology of non-immune hydrops fetalis: an update. Am J Med Genet A. 2015;167A:1082-1088.

10. Williams IA, Kleinman CS. Is hydrops fetalis a manifestation of fetal pulmonary edema caused by impaired lymphatic drainage? Ultrasound Obstet Gynecol. 2008;31:96-99.

11. Ismail KMK, Martin WL, Ghosh S, Whittle MJ, Kilby MD. Etiology and outcome of hydrops fetalis. J Matern Fetal Med. 2001;10:175-181.

12. American College of Obstetricians and Gynecologists Committee on Genetics Committee Opinion No. 581: the use of chromosomal microarray analysis in prenatal diagnosis. Obstet Gynecol. 2013;122:1374-1377.
13. Gimovsky AC, Luzi $P$, Berghella $V$. Lysosomal storage disease as an etiology of nonimmune hydrops. Am J Obstet Gynecol. 2015;212:281-290.

14. Kooper AJA, Janssens PMW, de Groot ANJA, et al. Lysosomal storage diseases in non-immune hydrops fetalis pregnancies. Clin Chim Acta. 2006;371:176-182.

15. Hellmund A, Berg C, Geipel A, Muller A, Gembruch U Prenatal diagnosis of fetal akinesia deformation sequence (FADS): a study of 79 consecutive cases. Arch Gynecol Obstet. 2016;294:697-707. [Epub ahead of print]

16. Bellini C, Mazzella M, Arioni C, et al. Hennekam syndrome presenting as nonimmune hydrops fetalis, congenital chylothorax, and congenital pulmonary lymphangiectasia. Am J Med Genet A. 2003;120A: 92-96.

17. Ostergaard P, Simpson MA, Connell FC, et al. Mutations in GATA2 cause primary lymphedema associated with a predisposition to acute myeloid leukemia (Emberger syndrome). Nat Genet. 2011;43:929-931.

18. Burin MG, Scholz AP, Gus R, et al. Investigation of lysosomal storage diseases in nonimmune hydrops fetalis. Prenat Diagn. 2004;24:653-657.

19. Carvalho S, Martins M, Fortuna A, Ramos U, Ramos C, Rodrigues MC. Galactosialidosis presenting as nonimmune fetal hydrops: a case report. Prenat Diagn. 2009:29:895-896.

20. Cheng Y, Verp MS, Knutel T, Hibbard JU. Mucopolysaccharidosis type VII as a cause of recurrent non-immune hydrops fetalis. J Perinat Med. 2003;31:535-537.

21. Whybra C, Mengel E, Russo A, et al. Lysosomal storage disorder in nonimmunological hydrops fetalis (NIHF)-more common than assumed? Report of four cases with transient NIHF and review of the literature. Orphanet J Rare Dis. 2012:7:86-95.

22. Verma J, Thomas DC, Sharma S, et al. Inherited metabolic disorders: prenatal diagnosis of lysosomal storage disorders. Prenat Diagn. 2015;35:1137-1147.

23. Surmeli-Onay O, Yakarisik S, Korkmaz A, et al. Prenatal-onset NiemannPick type $C$ disease in nonimmune hydrops fetalis. Pediatr Neonatol. 2013;54:344-347.

24. Swarr DT, Khalek N, Treat J, et al. Expanding the differential diagnosis of fetal hydrops: an unusual presentation of megalencephaly-capillary malformation syndrome. Prenat Diagn. 2013;33:1010-1012.

25. Terespolsky D, Farrell SA, Siegel-Bartelt J, Weksberg R. Infantile lethal variant of Simpson-Golabi Behmel syndrome associated with hydrops fetalis. Am J Med Genet. 1995;59:329-333.

26. Datkhaeva I, Arboleda VA, Senaratne TN, Nikpour G, Meyerson C, Geng $Y$, et al. Identification of novel PIEZO1 variants using prenatal exome sequencing and correlation to ultrasound and autopsy findings of recurrent hydrops fetalis. Am J Med Genet. 2018 Sept 23; doi:10.1002/ ajmg.a.40533 [Epub ahead of print].

27. Fotiou E, Martin-Almedina S, Simpson MA, Lin S, Gordon K, Brice G. et al. Novel mutations in PIEZO1 cause an autosomal recessive generalized lymphatic dysplasia with non-immune hydrops fetalis. Nat Commun. 2015;6:8085.

28. Takci S, Gharibzadeh M, Yurdakok M, et al. Etiology and outcome of hydrops fetalis: report of 62 cases. Pediatr Neonatol. 2014;55: 108-113. 